\title{
El derecho a percibir la "compensación por comida" correspondiente a los días teletrabajados durante la pandemia.
}

\author{
Carmen Viqueira Pérez \\ Catedrática de Derecho del Trabajo y de la Seguridad Social. Universidad de Alicante
}

Resumen: El teletrabajo impuesto durante la pandemia plantea una peculiar problemática en torno a la variada gama de "ayudas de comedor" que con frecuencia implementan los convenios colectivos para los trabajadores que realizan jornada partida, cuyo eventual devengo durante aquellas semanas resulta muy dudoso. Esta es la cuestión sobre la que reflexiona la sentencia comentada.

Palabras clave: Jornada partida. Teletrabajo. Tiket comida. Pandemia.

Abstract: Telework imposed during the pandemic poses a peculiar problem around the varied range of "meal aids" that frequently implement collective agreements for split day workers, whose eventual accrual during those weeks it is very doubtful. This is the question on which reflects on the commented sentence.

Keywords: Split day work. Telework. Meal aids. Pandemic.

\section{Introducción}

La crisis sanitaria derivada del COVID-19 originó un forzado crecimiento exponencial del teletrabajo a distancia. Aunque puede observarse alguna variación en los datos dependiendo de la fuente que los proporciona, a trazo grueso puede afirmarse que en 2019 solo un $4,8 \%$ de los ocupados trabajaba desde casa normalmente o más de la mitad de los días, en tanto que, durante los meses de restricción de la movilidad, el porcentaje de trabajadores que prestaban sus servicios a distancia con soporte de las TIC's se incrementó hasta alcanzar el 34\% del total.

Basta conocer este dato (y recordar la experiencia que todos hemos vivido) para comprender el desajuste que, inevitablemente y en más de un aspecto, mostró el marco normativo (tanto el legal como el convencional) con respecto a la realidad que había de ordenar. Buen ejemplo de ello nos lo proporciona la problemática planteada en torno a la variada gama de "ayudas de comedor" que con frecuencia implementan los convenios colectivos para los trabajadores que realizan jornada partida, cuyo eventual devengo durante las semanas en las que se impuso el teletrabajo COVID resulta dudoso. Esta es la cuestión sobre la que reflexiona la sentencia comentada.

\section{Identificación de la resolución judicial comentada}

Tipo de resolución judicial: sentencia.

Órgano judicial: Audiencia Nacional. Sala de lo Social 
Número de resolución judicial y fecha: Sentencia núm. 196/2021, de 22 de septiembre.

Tipo y número recurso o procedimiento: Conflicto colectivo. Número de recurso $47 / 2021$

ECLI:ES:AN:2021:3818.

Fuente: CENDOJ.

Ponente: Ilmo. Sr. D. José Luis Niño Romero.

Votos Particulares: Cuenta con un voto particular.

\section{Problema suscitado. Hechos y antecedentes}

La sentencia dilucida si la "compensación por comida" prevista en el convenio colectivo para quienes trabajan en régimen de jornada partida debió seguir devengándose también en la situación de teletrabajo que tuvo lugar durante la pandemia o si, por el contrario, el devengo de la "compensación por comida" no puede únicamente ligarse al hecho de que el trabajo se preste en régimen de jornada partida. Se trata de determinar, en fin, si los trabajadores de la empresa con jornada partida, tenían derecho a seguir percibiendo aquella ayuda durante los días en que, a consecuencia de la declaración del estado de alarma, pasaron a prestar servicios desde su domicilio.

Durante el estado de alarma decretado a raíz del COVID, la empresa UMIVALE (una Mutua de Accidentes de Trabajo y Enfermedades Profesionales) implantó la posibilidad de realizar la actividad en régimen de teletrabajo en aquellos departamentos que no se vieron obligados a mantener una actividad presencial esencial. Los trabajadores que prestaron su trabajo en remoto desde sus domicilios realizaron su actividad en las mismas condiciones que ordenaban su prestación cuando ésta era presencial y se cumplía en el centro de trabajo, de modo que debían estar disponibles en el horario laboral establecido (jornada partida) pudiendo la empresa comprobar el cumplimiento de este régimen a través de diversos mecanismos (conexiones a los aplicativos informáticos, desvió de teléfonos...).

Pese a que los trabajadores a jornada partida mantuvieron este régimen cuando, por decisión de la empresa, pasaron a "situación de teletrabajo", dejaron de percibir la "compensación por comida" que la empresa únicamente abonó a los trabajadores presenciales. En este contexto, la sección sindical de UGT elevó consulta a la Comisión Paritaria del Convenio por entender que, dado que el teletrabajo se prestó en régimen de jornada partida, los teletrabajadores devengaron la compensación por comidas. El silencio de la Comisión Paritaria origina la interposición del conflicto colectivo (al que CCOO se adhiere) que la sentencia resuelve desestimando la pretensión.

\section{Posición de las partes}

El sindicato entiende que el Convenio colectivo no condiciona el derecho a percibir la "compensación por comida" a que la prestación de servicios se haga de forma presencial, sino únicamente a que exista una prestación efectiva de servicios, de modo que siempre que el tiempo de interrupción de la jornada para comer se encuentre dentro de los márgenes que señala el convenio (que no sea inferior a una hora ni superior a dos), se tendrá derecho a una "compensación por comida" en los términos fijados en su artículo 41, no siendo relevante que la prestación se lleve a cabo desde el domicilio del trabajador (más exactamente "en" el domicilio). Sostiene, en fin, que mantener lo contrario implicaría otorgar a los teletrabajadores un trato distinto a los trabajadores presenciales que infringiría el mandato de igualdad contenido en el art. 20 del convenio ("La igualdad de derechos legales y convencionales de los teletrabajadores respecto a los trabajadores comparables que trabajan en las mismas instalaciones de la empresa") y en la "Declaración relativa al teletrabajo" de 10 de 
febrero de 2015 ("Por lo que se refiere a las condiciones laborables, los teletrabajadores tendrán los mismos derechos consagrados en la legislación y en los convenios colectivos aplicables, al igual que los trabajadores de las categorías equiparables que trabajan en centro de trabajo de la empresa").

Por su parte, la empresa sostiene que la "compensación por comida" tiene por finalidad compensar, a los trabajadores que prestan su trabajo en régimen de jornada partida, el mayor gasto que se deriva de la necesidad por comer fuera de su domicilio, de modo que, cuando el trabajador presta su trabajo desde su propio domicilio, la "compensación por comida" carece de sentido. Añade que, conforme a esta interpretación ha actuado siempre la empresa, para la que la prestación de trabajo en remoto es enteramente excepcional -con anterioridad a la pandemia y también tras ella- por lo que únicamente permite prestar servicios en régimen de teletrabajo como una concesión extraordinaria por motivos relacionados con la salud o con el cuidado de familiares, sin abonar la "compensación por comidas".

\section{Normativa aplicable al caso}

La cuestión debatida pende, en primer término, de la interpretación de lo establecido en el convenio colectivo estatal de seguros, reaseguros y mutuas colaboradoras de la Seguridad Social (publicado en el BOE de 1 de junio de 2017) con respecto a la compensación por comida por jornada partida (art. 41) y con respecto a la regulación de la jornada partida (art.47.9).

Art. 41: "La compensación por comida regulada en el artículo 47 del Convenio por jornada partida, equivaldrá como mínimo a los importes que a continuación se indican para los años de vigencia del Convenio: Año 2016 importe que figura en el anexo III para tal concepto; año 2017 10,90 euros; año 201811 euros y año 2019 11,10 euros. Para el año 2017 el importe indicado resultará aplicable desde el día 1 de mayo. En el artículo 47 se regula la jornada laboral y su distribución, dedicándose el apartado 9.A a la jornada partida que dispone lo siguiente:

Art. 47.9. A) "Jornada partida. La distribución horaria de la jornada partida se realizará conforme a las siguientes pautas y criterios:

a) Las horas de referencia para la entrada y salida del trabajo serán las 8 y las 17,30 horas, respectivamente, con la posibilidad de aplicar una flexibilidad horaria a partir de la hora de entrada y de salida de hasta 60 minutos.

b) El tiempo de comida no podrá ser inferior a una hora ni superior a dos.

c) Siempre que el tiempo para comida se encuentre dentro de estos márgenes, se tendrá derecho a una compensación por comida en los términos fijados en el artículo $41 \mathrm{del}$ presente Convenio, por día trabajado en jornada partida, procediendo su entrega salvo que la empresa esté facilitando a su cargo servicio de restaurante o comedor en el centro de trabajo o sus cercanías inmediatas, o salvo que se hubieran pactado otras compensaciones equivalentes que, por su razón de ser o naturaleza, se hubieran originado para compensar este concepto por jornada partida.

La empresa podrá sustituir las fórmulas indirectas de prestación del servicio de comedor que pudieran concurrir por entrega dineraria del importe señalado anteriormente.

d) Atendiendo siempre a las características organizativas y funcionales de la Empresa, se tenderá a establecer la jornada continuada de 8 a 15 horas para el período estival (de 15 de junio a 15 de septiembre), así como la libranza de todos los sábados del año.

e) Se establece, con carácter general, la jornada continuada en horario de mañana para todos los viernes del año. 
Se exceptúan, en todo caso, los "Supuestos especiales», que se regulan en el artículo $48 \mathrm{del}$ presente Convenio, así como las "Peculiaridades de las M.C.S.S.» reguladas en el artículo 50 del mismo"

Por lo que se refiere a la igualdad de derechos de los teletrabajadores y los trabajadores presenciales, ha de considerarse lo señalado en el convenio (art. 20) y lo establecido en la "Declaración relativa al teletrabajo" de 10 de febrero de 2015 (apartado "condiciones de empleo).

Art. 20 del convenio: que establece como criterio a aplicar en el teletrabajo "La igualdad de derechos legales y convencionales de los teletrabajadores respecto a los trabajadores comparables que trabajan en las mismas instalaciones de la empresa".

"Declaración relativa al teletrabajo": "Por lo que se refiere a las condiciones laborables, los teletrabajadores tendrán los mismos derechos consagrados en la legislación y en los convenios colectivos aplicables, al igual que los trabajadores de las categorías equiparables que trabajan en centro de trabajo de la empresa"

\section{Parte dispositiva}

La sentencia afirma que una interpretación integradora de lo señalado en el Convenio Colectivo conduce a concluir que la "compensación por comida" está contemplada para la realización de la jornada partida presencial pero no en teletrabajo" ya que no existe para la empresa la obligación de "realizar el abono de una cantidad de dinero, sino que (...) procede la entrega de la cantidad fijada "salvo que" la empresa esté facilitando a su cargo un servicio de restaurante o comedor en el centro de trabajo". Por otro lado, el hecho de que la prestación del servicio de comedor se lleve a cabo "en el centro de trabajo o en sus cercanías inmediatas" conduce a considerar que "el sentido que debe darse a la norma discutida es el postulado por la parte demandada, esto es, que la compensación por comida en jornada partida está pensada para el trabajo presencial y no para el teletrabajo". Afirma, además, que "en el presente caso no se parte de la misma situación entre los trabajadores presenciales y los no presenciales y por ello no se puede pretender un trato idéntico, pues éstos no deben realizar desplazamiento alguno en la jornada partida, lo que si deben realizar los presenciales". Y concluye que, por todo ello, ha de desestimarse la demanda.

\section{Pasajes decisivos}

El hilo argumental de la sentencia arranca recordando que la correcta interpretación de los preceptos de los convenios colectivos está sometida a las reglas de interpretación de las normas y de los contratos, como ha señalado la jurisprudencia: "En este sentido se ha pronunciado la Sala IV del Tribunal Supremo, en sentencia de 10 de noviembre de 2016 (Rec. 290/2015) -entre otras- que resume su reiterada doctrina en los siguientes términos: "hemos señalado que: a) el carácter mixto del Convenio Colectivo -norma de origen convencional/contrato con eficacia normativa- determina que su interpretación ha de atender tanto a las reglas legales atinentes a la hermenéutica de las normas jurídicas, como a aquellas otras que disciplinan la interpretación de los contratos, esto es, los Arts. 3 , 4 y 1.281 y 1.289 del CC ; b) la interpretación de un Convenio Colectivo ha de combinar los criterios de orden lógico, gramatical e histórico, junto con el principal de atender a las palabras e intenciones de los contratantes; c) las normas de interpretación de los Arts. 1.282 y siguientes del CC tiene carácter de subsidiariedad en su aplicación, de forma que cuando la literalidad de las cláusulas de un contrato sean claras, no son de aplicación otras diferentes que las correspondientes al sentido gramatical; o dicho de otro modo, el Art. 1.281 del CC consta de dos párrafos, que persiguen la doble finalidad de evitar que se tergiverse lo que aparece claro, o que se admita, sin aclarar lo que se ofrezca oscuro, siendo factor decisivo de interpretación, en el primer supuesto las palabras empleadas, y en el segundo la intención evidente de los contratantes. En el mismo sentido, entre otras muchas, la sentencia del Tribunal Supremo, Sala Cuarta, de 20 de julio de 2016 (Rec. 197/2015)". 
Partiendo de esta base, entra en la interpretación de la regulación convencional, indicando que: "Una primera lectura del precepto podría dar a entender que basta con trabajar en jornada partida para devengar la compensación, si bien la redacción de la letra c) contiene además otras previsiones que hacen necesaria una interpretación integradora del precepto en su conjunto, lo que nos lleva a entender que la compensación está contemplada para la realización de la jornada partida presencial pero no en teletrabajo. A la conclusión anterior se llega si se tiene en cuenta que es al empresario al que corresponde la concreta forma de materialización de la prestación compensatoria, esto es, si la realiza mediante un servicio de comedor en el centro de trabajo o bien mediante su pago en metálico. El precepto no obliga a realizar el abono de una cantidad de dinero, sino que señala que procede la entrega de la cantidad fijada en el artículo 41 del Convenio "salvo que" la empresa esté facilitando a su cargo un servicio de restaurante o comedor en el centro de trabajo. A renglón seguido se afirma que la empresa "podrá sustituir" las fórmulas indirectas de prestación del servicio de comedor por entrega dineraria del importe señalado, de lo que se deduce que la regla general es la prestación en especie a través de un servicio de comedor y la excepción el abono en metálico. Por otra parte la prestación del servicio de comedor es en el centro de trabajo o en sus cercanías inmediatas, por lo que el sentido que debe darse a la norma discutida es el postulado por la parte demandada, esto es, que la compensación por comida en jornada partida está pensada para el trabajo presencial y no para el teletrabajo".

Esta interpretación integradora, afirma la sentencia, no resulta contraria a la igualdad de trato entre trabajadores presenciales y no presenciales establecida en el art. 20 del convenio colectivo porque: "En el presente caso no se parte de la misma situación entre los trabajadores presenciales y los no presenciales y por ello no se puede pretender un trato idéntico, pues éstos no deben realizar desplazamiento alguno en la jornada partida, lo que si deben realizar los presenciales".

\section{Comentario}

La crisis sanitaria derivada del COVID originó un forzado y exponencial exponencial crecimiento del teletrabajo a distancia. Aunque puede observarse alguna variación en los datos dependiendo de la fuente que los proporciona, a trazo grueso puede afirmarse que en 2019 solo un $4,8 \%$ de los ocupados trabajaba desde casa normalmente o más de la mitad de los días, en tanto que, durante los meses de restricción de la movilidad, el porcentaje de trabajadores que prestaban sus servicios a distancia con soporte de las TIC's se incrementó hasta alcanzar el 34\% del total.

Basta conocer este dato (y recordar la experiencia que todos hemos vivido) para comprender el desajuste que, inevitablemente y en más de un aspecto, mostró el marco normativo con respecto a la realidad que había de ordenar. Buen ejemplo de ello nos lo proporciona la problemática planteada en torno a la variada gama de "ayudas de comedor" que con frecuencia implementan los convenios colectivos para los trabajadores que realizan jornada partida, cuyo eventual devengo durante las semanas en las que se impuso el teletrabajo COVID resulta muy dudoso. Esta es la cuestión sobre la que reflexiona la sentencia comentada al interpretar el alcance de las previsiones que el convenio colectivo contiene a este respecto.

En efecto, el convenio colectivo aplicable en la empresa (estatal de seguros, reaseguros y mutuas colaboradoras de la Seguridad Social) establece que los trabajadores que prestan sus servicios con jornada partida con una pausa no inferior a una hora ni superior a dos tendrán derecho a una "compensación por comida (...) salvo que la empresa esté facilitando a su cargo servicio de restaurante o comedor" en el propio centro de trabajo o en sus cercanías (y excepción hecha de que la empresa hubiera "pactado otras compensaciones equivalentes") y esta previsión es susceptible de ser interpretada en distintos sentidos.

Puede entenderse -en una lectura pegada a la literalidad del texto- que el convenio establece la obligatoriedad de abonar esta "compensación por comida" a todos los trabajadores que presten su trabajo en régimen de jornada partida, salvo que la 
empresa facilite directamente el servicio de restaurante o comedor; de modo que el hecho de que la jornada partida se preste en los locales de la empresa o en el domicilio del trabajador resultaría ser un dato irrelevante. Y puede -en una lectura contextualizada del precepto- sostenerse que el abono de la "compensación por comida" procede únicamente en el escenario de la presencialidad porque la previsión del convenio -ya sea en su modalidad de ofrecer servicio de comedor, ya sea en su modalidad de abonar compensación- es una medida finalista, que tiene por objeto mitigar el mayor gasto económico que cabe presumir que asume el trabajador que -por prestar el trabajo en jornada partida- ha de comer fuera de casa y esa circunstancia no concurre cuando el trabajador trabaja en su domicilio.

Defienden la primera posición el sindicato que promueve el conflicto colectivo y el voto particular discrepante que, además, refuerzan su postura alegando que circunscribir el reconocimiento de la "compensación por comida" a los trabajadores presenciales y negarlo a los que teletrabajan infringe el mandato de igualdad que establece el propio convenio en su artículo 20 que, al regular el teletrabajo, señala que "para la realización y puesta en práctica del teletrabajo en la empresa, resulta oportuno establecer los siguientes criterios (...) la igualdad de derechos legales $y$ convencionales de los teletrabajadores respecto a los trabajadores comparables que trabajan en las mismas instalaciones de la empresa".

La sentencia, sin embargo, se inclina por la posición contraria y, tras recordar las reglas que han de aplicarse en la interpretación de los convenios colectivos, entiende que ha de hacerse una interpretación "integradora" del precepto en su conjunto que conduciría a considerar que el derecho a percibir esta compensación se anuda a la realización de la jornada continuada presencial "pero no en teletrabajo".

A mi juicio, en efecto, ello es así. De entrada, me parece innegable la necesidad de hacer una interpretación del precepto que vaya más allá de su literalidad (que, por otro lado, tampoco es en exceso concluyente) y permita darle un sentido acorde con el resto de la herramienta normativa. $Y$ esa interpretación integradora apunta a considerar que las medidas que a este respecto contiene el convenio (prestar servicio de comedor, abonar la "compensación por comida") atiende a la situación de quienes prestan sus servicios en jornada partida y en un régimen (pausa mínima de 1 hora y máxima de 2 horas) que les imposibilita desplazarse a su domicilio para comer y regresar a su puesto de trabajo. Por otro lado, el modo en que el convenio articula estas medidas conduce a pensar que no tiene la empresa la obligación de abonar la "compensación por comida" a todos los trabajadores que prestan servicios en régimen de jornada partida, sino que lo que el convenio establece es que procede este abono cuando la empresa no haya optado por ofrecer un servicio de comedor en el centro de trabajo o en sus alrededores; esto es, como prestación subsidiaria de la obligación de ofertar el servicio de comedor. Y, finalmente, el hecho de que la empresa pueda cumplir su obligación ofertando este servicio de comedor en el centro de trabajo o en lugar cercano a éste revela que la idea del precepto es atender a la situación de los trabajadores que prestan trabajo presencialmente (de ahí que el comedor deba situarse cerca del centro de trabajo).

En este contexto, como también señala la sentencia, la situación de los trabajadores presenciales es radicalmente distinta de la situación en que se encuentran los teletrabajadores -dado que estos últimos no se ven perjudicados por el hecho de tener que comer fuera de sus domicilios- $y$, por ello, este trato distinto no afecta al mandato de igualdad que expresamente contiene el artículo 20 del convenio.

\section{Apunte final}

Como se señalaba al principio, la situación de teletrabajo COVID se desenvuelve en un marco normativo que muestra su desajuste con respecto a la realidad que se ve compelido a regular. Es lógico que ello sea así y es lógico que estas cuestiones acaben siendo resueltas en el ámbito jurisdiccional. Ello no obstante, parte de la litigiosidad que de esta situación se deriva, puede obviarse con un funcionamiento adecuado de otras instancias y, en este sentido y por lo que se refiere al litigio que la 
sentencia resuelve, llama poderosamente la atención la falta de respuesta de la Comisión Mixta del convenio a la que -en primer término- se dirige el sindicato accionante y que bien pudiera haber evitado el planteamiento del conflicto colectivo. 